Vietnam Journal of Mechanics, VAST, Vol.38, No. 1 (2016), pp. $63-79$

DOI:10.15625/0866-7136/38/1/7036

\title{
THERMOMECHANICAL POSTBUCKLING OF THICK FGM PLATES RESTING ON ELASTIC FOUNDATIONS WITH TANGENTIAL EDGE CONSTRAINTS
}

\author{
Hoang Van Tung \\ Hanoi Architectural University, Vietnam \\ *E-mail: inter0105@gmail.com \\ Received September 17, 2015
}

\begin{abstract}
This paper investigates the effects of tangential edge constraints and elastic foundations on the buckling and postbuckling behavior of thick FGM rectangular plates resting on elastic foundations and subjected to thermal and thermomechanical loading conditions. Material properties are assumed to be temperature dependent, and graded in the thickness direction according to a simple power law distribution in terms of the volume fractions of constituents. Governing equations are based on the higher order shear deformation plate theory incorporating the von Karman geometrical nonlinearity, initial geometrical imperfection, tangential edge constraints and Pasternak type elastic foundations. Approximate solutions are assumed to satisfy simply supported boundary conditions and Galerkin procedure is applied to derive expressions of buckling loads and load-deflection relations. In thermal postbuckling analysis, an iteration algorithm is employed to determine critical buckling temperatures and postbuckling temperaturedeflection equilibrium paths. The separate and simultaneous effects of tangential edge restraints, elastic foundations and temperature dependence of material properties on the buckling and postbuckling responses of higher order shear deformable FGM plates are analyzed and discussed.
\end{abstract}

Keywords: Functionally graded materials, tangential edge constraint, temperature dependent property, buckling and postbuckling, elastic foundations.

\section{INTRODUCTION}

Functionally graded materials (FGMs) are microscopically composites usually composed from a mixture of metal and ceramic constituents and have some advanced features in comparison with conventional laminated composites. As a result, the stability of FGM structures is an attractive topic for many researchers. Linear buckling of simply supported FGM rectangular plates subjected to compressive and thermal loads has been investigated by Eslami and his co-authors [1-4] and Lanhe [5] using classical, first order and higher order shear deformation theories and an analytical method. Thermal and

(C) 2016 Vietnam Academy of Science and Technology 
mechanical linear buckling of FGM plates have also been investigated using numerical methods in works by Zhao et al. [6], Nguyen and his collaborators [7-9] and by Liew and his co-authors [10-12]. Tung and Duc [13,14] employed Galerkin method on the basis of classical and higher order shear deformation theories to investigate nonlinear stability of simply supported FGM plates subjected to mechanical and thermal loading conditions with and without elastic foundations and temperature independent properties. Nonlinear stability and postbuckling of FGM plates have also been addressed in some studies by Woo et al. [15] utilizing an analytical approach, by Shen [16] making use of a two-step perturbation technique and Lee et al. [17] using the element-free kp-Ritz method. Buckling and postbuckling of FGM sandwich plates consisting of homogeneous and FGM layers have been analyzed in some works by Zenkour [18], Zenkour and Sobhy [19] employing an analytical method and by Shen and Li [20] and Wang and Shen [21] basing on an semi-analytical approach.

In foregoing studies, only two extreme cases of in-plane constraints of edges have been considered. Specifically, the edges of plates are usually assumed to be either unrestrained (free movable edges) or fully restrained (immovable edges). However, in practical situations, the tangential motion of the edges may be partially restrained only. This results from the boundary supports are not completely rigid and they can deform elastically. Librescu et al. [22,23] analyzed the effects of tangential edge constraints on the postbuckling and vibration of laminated flat and curved panels subjected to mechanical and thermal loads. These works indicated that, in the static case, the degree of the tangential edge restraint can has great effects on the behavior of plates and shells. In spite of considerable effects of tangential edge constraints and increasing use of FGMs, studies on this subject are comparatively scarce. Recently, the author used an analytical approach based on the classical thin shell theory to analyze the postbuckling behavior of thin FGM cylindrical panels and circular cylindrical shells subjected to mechanical and thermal loads taking the effects of tangential edge constraints into consideration [24,25] without and with temperature dependent material properties, respectively. It was shown in work [25] that the effects of temperature dependence of material properties on load carrying capacity become more pronounced and deteriorative for partially movable edge FGM cylindrical shells.

This paper extends previous works $[14,24]$ to investigate separate and simultaneous influences of tangential edge constraints and temperature dependent material properties on the buckling and postbuckling behavior of higher order shear deformable FGM rectangular plates resting on elastic foundations and subjected to thermal and thermomechanical loads. The novelty of the present study in comparison with works $[14,24]$ results from temperature dependence of material properties and varying degree of tangential edge constraint. Formulations are based on the higher order shear deformation plate theory taking von Karman geometrical nonlinearity, initial geometrical imperfection, Pasternak type elastic foundations and tangential edge restraints into consideration. Approximate solutions of deflection and stress function are assumed to satisfy simply supported boundary conditions and Galerkin method is employed to determine expressions of buckling loads and load-deflection relations. In thermal postbuckling analysis, 
an iteration algorithm is adopted to obtain critical buckling temperatures and postbuckling temperature-deflection equilibrium paths. The effects played by the degree of the tangential edge constraints, temperature dependent material properties, stiffness of elastic foundations and imperfection on the buckling and postbuckling behavior of thick FGM plates are analyzed and discussed.

\section{FGM RECTANGULAR PLATE ON AN ELASTIC FOUNDATION}

Consider an FGM rectangular plate of plan-form dimensions $a$ and $b$, and uniform thickness $h$ resting on an elastic foundation. The plate is made from a mixture of ceramics and metals, and is defined in a coordinate system $(x, y, z)$ whose origin is located at the corner on the middle surface of the plate, $x$ and $y$ are in-plane coordinates towards edges $a$ and $b$, respectively, and $z$ is perpendicular to the middle surface $(-h / 2 \leq z \leq h / 2)$ as shown in Fig. 1.

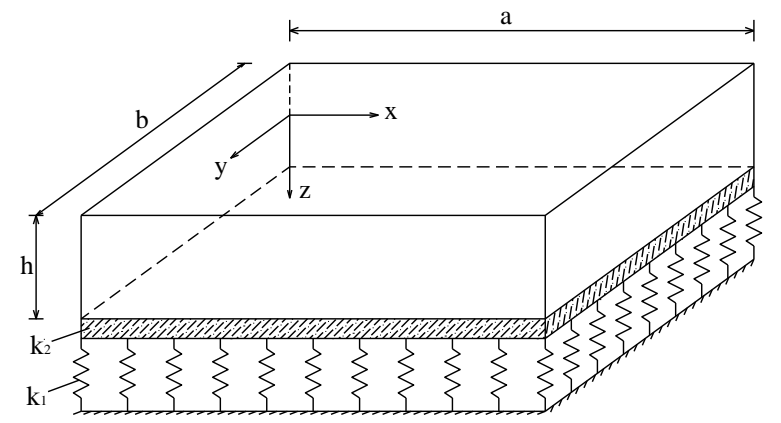

Fig. 1. Geometry and coordinates system of an FGM rectangular plate on an elastic foundation

Suppose that the material composition of the plate varies smoothly along the thickness in such a way that the bottom surface is metal-rich and the top surface is ceramic-rich by following a simple power law in terms of the volume fractions of the constituents as

$$
V_{m}(z)=\left(\frac{2 z+h}{2 h}\right)^{N}, \quad V_{c}(z)=1-V_{m}(z)
$$

where $V_{m}$ and $V_{c}$ are the volume fractions of metal and ceramic constituents, respectively, and $N \geq 0$ is volume fraction index.

Practically, FGMs are most commonly used in high temperature environments, and significant changes in material properties are inherent. Usually, the elasticity modulus decreases, and the thermal expansion coefficient increases at elevated temperatures. Therefore, it is essential to account for this temperature dependence for accurate and reliable prediction of the response of thermally loaded FGM structures.

It is assumed that the effective properties $\operatorname{Pr}_{e f f}$ of FGM plates change only in the thickness direction $z$ and can be determined by the linear rule of mixture as

$$
\operatorname{Pr}_{e f f}(z, T)=\operatorname{Pr}_{m}(T) V_{m}(z)+\operatorname{Pr}_{c}(T) V_{c}(z),
$$


where Pr denotes a specific material property assumed to be temperature-dependent in the present study, and subscripts $m$ and $c$ represent the metal and ceramic constituents, respectively.

From Eqs. (1) and (2) the effective properties of FGM plates such as the modulus of elasticity $E$ and the coefficient of thermal expansion $\alpha$ can be written in the form

$$
\begin{aligned}
& E(z, T)=E_{c}(T)+E_{m c}(T)\left(\frac{2 z+h}{2 h}\right)^{N}, \\
& \alpha(z, T)=\alpha_{c}(T)+\alpha_{m c}(T)\left(\frac{2 z+h}{2 h}\right)^{N},
\end{aligned}
$$

where

$$
E_{m c}(T)=E_{m}(T)-E_{c}(T), \quad \alpha_{m c}(T)=\alpha_{m}(T)-\alpha_{c}(T),
$$

and Poisson's ratio $v$ is assumed to be constant. In the present study, the FGM plate is fully rested on an elastic foundation and the FGM plate-foundation interaction is represented by Pasternak model as

$$
q_{f}=k_{1} w-k_{2} \Delta w
$$

where $\Delta=\partial^{2} / \partial x^{2}+\partial^{2} / \partial y^{2}$ is Laplace operator, $w$ is the deflection (transverse displacement) of the plate; $k_{1}$ is Winkler foundation modulus and $k_{2}$ is the shear layer foundation stiffness of Pasternak model.

\section{THEORETICAL FORMULATIONS}

In the present study, Reddy's higher order shear deformation plate theory [26] is used to establish governing equations for buckling and postbuckling analysis of thick FGM plates. Based on this theory, normal strains $\varepsilon_{x}, \varepsilon_{y}$, in-plane shear strain $\gamma_{x y}$ and transverse shear deformations $\gamma_{x z}, \gamma_{y z}$ are represented as

$$
\left(\begin{array}{c}
\varepsilon_{x} \\
\varepsilon_{y} \\
\gamma_{x y}
\end{array}\right)=\left(\begin{array}{c}
\varepsilon_{x}^{0} \\
\varepsilon_{y}^{0} \\
\gamma_{x y}^{0}
\end{array}\right)+z\left(\begin{array}{c}
k_{x}^{1} \\
k_{y}^{1} \\
k_{x y}^{1}
\end{array}\right)+z^{3}\left(\begin{array}{c}
k_{x}^{3} \\
k_{y}^{3} \\
k_{x y}^{3}
\end{array}\right), \quad\left(\begin{array}{c}
\gamma_{x z} \\
\gamma_{y z}
\end{array}\right)=\left(\begin{array}{c}
\gamma_{x z}^{0} \\
\gamma_{y z}^{0}
\end{array}\right)+z^{2}\left(\begin{array}{c}
k_{x z}^{2} \\
k_{y z}^{2}
\end{array}\right),
$$

where

$$
\begin{aligned}
& \left(\begin{array}{c}
\varepsilon_{x}^{0} \\
\varepsilon_{y}^{0} \\
\gamma_{x y}^{0}
\end{array}\right)=\left(\begin{array}{c}
u_{, x}+w_{, x}^{2} / 2+w_{, x} w_{, x}^{*} \\
v_{, y}+w_{, y}^{2} / 2+w_{, y}^{*} w_{, y}^{*} \\
u_{, y}+v_{, x}+w_{, x} w_{, y}+w_{, x}^{*} w_{, y}+w_{, x} w_{, y}^{*}
\end{array}\right),\left(\begin{array}{c}
k_{x}^{1} \\
k_{y}^{1} \\
k_{x y}^{1}
\end{array}\right)=\left(\begin{array}{c}
\phi_{x, x} \\
\phi_{y, y} \\
\phi_{x, y}+\phi_{y, x}
\end{array}\right), \\
& \left(\begin{array}{c}
k_{x}^{3} \\
k_{y}^{3} \\
k_{x y}^{3}
\end{array}\right)=-c_{1}\left(\begin{array}{c}
\phi_{y, y}+w_{, y y}+w_{, x y}^{*} \\
\phi_{x, y}+\phi_{y, x}+2 w_{, x y}+2 w_{, x y}^{*}
\end{array}\right), \\
& \left(\begin{array}{c}
\gamma_{x z}^{0} \\
\gamma_{y z}^{0}
\end{array}\right)=\left(\begin{array}{c}
\phi_{x}+w_{, x}+w_{x}^{*} \\
\phi_{y}+w_{, y}+w_{, y}^{*}
\end{array}\right), \quad\left(\begin{array}{c}
k_{x z}^{2} \\
k_{y z}^{2}
\end{array}\right)=-3 c_{1}\left(\begin{array}{c}
\phi_{x}+w_{, x}+w_{, x}^{*} \\
\phi_{y}+w_{, y}+w_{, y}^{*}
\end{array}\right),
\end{aligned}
$$

in which $c_{1}=4 /\left(3 h^{2}\right)$ and von Karman nonlinear terms are incorporated. Also, $u, v$ are displacement components along the $x, y$ directions, respectively, and $\phi_{x}, \phi_{y}$ are the 
rotations of normal to the midsurface with respect to $y$ and $x$ axes, respectively. Moreover, $w^{*}$ is a known function representing initial geometrical imperfection of the plate.

Hooke's law for an FGM plate is defined as

$$
\begin{aligned}
& \sigma_{x}=\frac{E(z, T)}{1-v^{2}}\left[\varepsilon_{x}+v \varepsilon_{y}-(1+v) \alpha(z, T) \Delta T\right], \\
& \sigma_{y}=\frac{E(z, T)}{1-v^{2}}\left[\varepsilon_{y}+v \varepsilon_{x}-(1+v) \alpha(z, T) \Delta T\right], \\
& {\left[\sigma_{x y}, \sigma_{x z}, \sigma_{y z}\right]=\frac{E(z, T)}{2(1+v)}\left[\gamma_{x y}, \gamma_{x z}, \gamma_{y z}\right],}
\end{aligned}
$$

where $\Delta T$ is temperature rise from thermal stress-free initial state, and is assumed to be independent of in-plane coordinates $x, y$. The force and moment resultants of the FGM plate are determined as

$$
\begin{aligned}
& \left(N_{i}, M_{i}, P_{i}\right)=\int_{-h / 2}^{h / 2} \sigma_{i}\left(1, z, z^{3}\right) d z, \quad i=x, y, x y \\
& \left(Q_{x}, K_{x}\right)=\int_{-h / 2}^{h / 2} \sigma_{x z}\left(1, z^{2}\right) d z, \quad\left(Q_{y}, K_{y}\right)=\int_{-h / 2}^{h / 2} \sigma_{y z}\left(1, z^{2}\right) d z .
\end{aligned}
$$

Introduction of Eqs. (6), (7) into Eqs. (8) and substitution of the resulting into Eqs. (9) give the constitutive relations as

$$
\begin{aligned}
& \left(N_{x}, M_{x}, P_{x}\right)=\frac{1}{1-v^{2}}\left[\left(E_{1}, E_{2}, E_{4}\right)\left(\varepsilon_{x}^{0}+v \varepsilon_{y}^{0}\right)+\left(E_{2}, E_{3}, E_{5}\right)\left(k_{x}^{1}+v k_{y}^{1}\right)\right. \\
& \left.+\left(E_{4}, E_{5}, E_{7}\right)\left(k_{x}^{3}+v k_{y}^{3}\right)-(1+v)\left(\Phi_{1}, \Phi_{2}, \Phi_{4}\right)\right], \\
& \left(N_{y}, M_{y}, P_{y}\right)=\frac{1}{1-v^{2}}\left[\left(E_{1}, E_{2}, E_{4}\right)\left(\varepsilon_{y}^{0}+v \varepsilon_{x}^{0}\right)+\left(E_{2}, E_{3}, E_{5}\right)\left(k_{y}^{1}+v k_{x}^{1}\right)\right. \\
& \left.+\left(E_{4}, E_{5}, E_{7}\right)\left(k_{y}^{3}+v k_{x}^{3}\right)-(1+v)\left(\Phi_{1}, \Phi_{2}, \Phi_{4}\right)\right], \\
& \left(N_{x y}, M_{x y}, P_{x y}\right)=\frac{1}{2(1+v)}\left[\left(E_{1}, E_{2}, E_{4}\right) \gamma_{x y}^{0}+\left(E_{2}, E_{3}, E_{5}\right) k_{x y}^{1}+\left(E_{4}, E_{5}, E_{7}\right) k_{x y}^{3}\right] \text {, } \\
& \left(Q_{x}, K_{x}\right)=\frac{1}{2(1+v)}\left[\left(E_{1}, E_{3}\right) \gamma_{x z}^{0}+\left(E_{3}, E_{5}\right) k_{x z}^{2}\right] \text {, } \\
& \left(Q_{y}, K_{y}\right)=\frac{1}{2(1+v)}\left[\left(E_{1}, E_{3}\right) \gamma_{y z}^{0}+\left(E_{3}, E_{5}\right) k_{y z}^{2}\right] \text {, }
\end{aligned}
$$


where

$$
\begin{aligned}
& \left(E_{1}, E_{2}, E_{3}, E_{4}, E_{5}, E_{7}\right)=\int_{-h / 2}^{h / 2} E(z, T)\left(1, z, z^{2}, z^{3}, z^{4}, z^{6}\right) d z, \\
& \left(\Phi_{1}, \Phi_{2}, \Phi_{4}\right)=\int_{-h / 2}^{h / 2} E(z, T) \alpha(z, T) \Delta T\left(1, z, z^{3}\right) d z,
\end{aligned}
$$

and specific expressions of temperature dependent coefficients $E_{i}=E_{i}(T)(i=1 \div 7)$ are analogous to those given in the [14] for case of temperature independent properties and are omitted here for sake of brevity.

Governing equations of higher order deformable FGM plates on elastic foundations have been derived in the [14]. Specifically, nonlinear equilibrium equation has the form

$$
\begin{aligned}
& c_{1}^{2}\left(D_{2} D_{5} / D_{4}-D_{3}\right) \Delta^{3} w+\left(c_{1} D_{2} / D_{4}+1\right) D_{6} \Delta^{2} w \\
& +\left(1-c_{1} D_{5} / D_{4}\right) \Delta\left[f_{, y y}\left(w_{, x x}+w_{, x x}^{*}\right)-2 f_{, x y}\left(w_{, x y}+w_{, x y}^{*}\right)+f_{, x x}\left(w_{, y y}+w_{, y y}^{*}\right)-k_{1} w+k_{2} \Delta w\right] \\
& -D_{6} / D_{4}\left[f_{, y y}\left(w_{, x x}+w_{, x x}^{*}\right)-2 f_{, x y}\left(w_{, x y}+w_{, x y}^{*}\right)+f_{, x x}\left(w_{, y y}+w_{, y y}^{*}\right)-k_{1} w+k_{2} \Delta w\right]=0,
\end{aligned}
$$

where

$$
\begin{aligned}
& D_{1}=\frac{E_{1} E_{3}-E_{2}^{2}}{E_{1}\left(1-v^{2}\right)}, \quad D_{2}=\frac{E_{1} E_{5}-E_{2} E_{4}}{E_{1}\left(1-v^{2}\right)}, \quad D_{3}=\frac{E_{1} E_{7}-E_{4}^{2}}{E_{1}\left(1-v^{2}\right)}, \\
& D_{4}=D_{1}-c_{1} D_{2}, \quad D_{5}=D_{2}-c_{1} D_{3}, \quad D_{6}=\frac{1}{2(1+v)}\left(E_{1}-6 c_{1} E_{3}+9 c_{1}^{2} E_{5}\right),
\end{aligned}
$$

and strain compatibility equation for an imperfect FGM plate is [14]

$$
\Delta f-E_{1}\left(w_{, x y}^{2}-w_{, x x} w_{, y y}+2 w_{, x y} w_{, x y}^{*}-w_{, x x} w_{, y y}^{*}-w_{, y y} w_{, x x}^{*}\right)=0 .
$$

In Eqs. (12) and (14), $f(x, y)$ is a stress function defined as

$$
N_{x}=f_{, y y}, \quad N_{y}=f_{, x x}, \quad N_{x y}=-f_{, x y} .
$$

In this study, the FGM plates are assumed to be simply supported at all edges. The associated boundary conditions are $[14,26]$

$$
\begin{array}{ll}
w=\phi_{y}=M_{x}=P_{x}=0, N_{x}=N_{x 0} & \text { at } x=0, a \\
w=\phi_{x}=M_{y}=P_{y}=0, N_{y}=N_{y 0} & \text { at } y=0, b
\end{array}
$$

In Eqs. (16) $N_{x 0}, N_{y 0}$ are prebuckling compressive force resultants at freely movable edges where $N_{x y}$ is zero-valued, and are fictitious compressive edge loads at tangentially restrained edges.

For the purpose of the present study, in-plane boundary conditions are assumed to be with varying degrees of tangential edge restraint. The degree of tangential edge restraint considered is bounded by the cases in which the tangential motion of the unloaded edges of a plate are either unrestrained or completely restrained, respectively, in the in-plane direction perpendicular to the plate edge. For two these cases, the plate 
edges are referred to as movable and immovable edges, respectively. All intermediate cases are referred to herein as partially movable edges and include elastically restrained edge constraints.

The average end-shortening displacement $\Delta_{1}$ between edges $x=0$ and $x=a$ is related to the corresponding fictitious compressive edge load $N_{x 0}$ by

$$
\Delta_{1} s_{1}=N_{x 0}
$$

where $s_{1}$ is the average tangential stiffness in the $x$ direction on each opposite edge. Similarly, for the edges $y=0$ and $y=b$, relation is expressed as

$$
\Delta_{2} s_{2}=N_{y 0}
$$

where $s_{2}$ is the average tangential stiffness in the $y$ direction on each opposite edge.

The expressions for the average end-shortening displacements are defined as [22, 23]

$$
\Delta_{1}=-\frac{1}{a b} \int_{0}^{a} \int_{0}^{b} \frac{\partial u}{\partial x} d y d x, \quad \Delta_{2}=-\frac{1}{a b} \int_{0}^{a} \int_{0}^{b} \frac{\partial v}{\partial y} d y d x
$$

Eqs. (17) and (18) indicate that values of $\Delta_{1}=0$ and $\Delta_{2}=0$ correspond to immovable edges at $x=0, a$ and $y=0, b$, respectively. These conditions are satisfied by selecting $s_{1} \rightarrow \infty$ and $s_{2} \rightarrow \infty$, respectively. In addition, values of $s_{1}=0$ and $s_{2}=0$ correspond to movable edges at $x=0, a$ and $y=0, b$, respectively. For these movable edge conditions, fictitious compressive edge loads are zero-valued, i.e. $N_{x 0}=0$ and $N_{y 0}=0$.

To satisfy boundary conditions (16), the approximate solutions are assumed as [14]

$$
\begin{aligned}
& \left(w, w^{*}\right)=(W, \mu h) \sin \beta_{m} x \sin \delta_{n} y, \\
& f=A_{1} \cos 2 \beta_{m} x+A_{2} \cos 2 \delta_{n} y+A_{3} \sin \beta_{m} x \sin \delta_{n} y+\frac{1}{2} N_{x 0} y^{2}+\frac{1}{2} N_{y 0} x^{2}, \\
& \phi_{x}=B_{1} \cos \beta_{m} x \sin \delta_{n} y, \quad \phi_{y}=B_{2} \sin \beta_{m} x \cos \delta_{n} y,
\end{aligned}
$$

where $\beta_{m}=m \pi / a, \delta_{n}=n \pi / b, W$ is amplitude of the deflection and $\mu$ is imperfection parameter. The coefficients $A_{i}(i=1 \div 3)$ are determined by substitution of Eqs. (20) and (21) into Eq. (14) as

$$
A_{1}=\frac{E_{1} \delta_{n}^{2}}{32 \beta_{m}^{2}} W(W+2 \mu h), \quad A_{2}=\frac{E_{1} \beta_{m}^{2}}{32 \delta_{n}^{2}} W(W+2 \mu h), \quad A_{3}=0 .
$$

Similarly, the coefficients $B_{1}, B_{2}$ are obtained as procedure described in the [14] as

$$
B_{1}=\frac{a_{12} a_{23}-a_{22} a_{13}}{a_{12}^{2}-a_{11} a_{22}} W, \quad B_{2}=\frac{a_{12} a_{13}-a_{11} a_{23}}{a_{12}^{2}-a_{11} a_{22}} W,
$$

where

$$
\begin{aligned}
&\left(a_{11}, a_{22}, a_{12}\right)=\left(c_{1}^{2} D_{3}+D_{1}-2 c_{1} D_{2}\right)\left(\beta_{m}^{2}, \delta_{n}^{2}, v \beta_{m} \delta_{n}\right) \\
&+\frac{1-v}{2}\left(c_{1}^{2} D_{3}+D_{1}-2 c_{1} D_{2}\right)\left(\delta_{n}^{2}, \beta_{m}^{2}, \beta_{m} \delta_{n}\right)+D_{6}(1,1,0), \\
&\left(a_{13}, a_{23}\right)=c_{1} D_{5}\left(\beta_{m}^{3}+\beta_{m} \delta_{n}^{2}, \delta_{n}^{3}+\delta_{n} \beta_{m}^{2}\right)-D_{6}\left(\beta_{m}, \delta_{n}\right) .
\end{aligned}
$$


Subsequently, Eqs. (20) and (21) are substituted into equilibrium equation (12) and applying Galerkin method for the resulting equation as procedure developed in the [14] yield

$$
\begin{gathered}
\left\{-\frac{\left(\bar{D}_{2} \bar{D}_{5}-\bar{D}_{3} \bar{D}_{4}\right)}{9 \bar{D}_{6} B_{h}^{6}}\right. \\
m n \pi^{8}\left(m^{2} B_{a}^{2}+n^{2}\right)^{3}+\frac{m n \pi^{6}}{16 B_{h}^{4}}\left(\frac{4}{3} \bar{D}_{2}+\bar{D}_{4}\right)\left(m^{2} B_{a}^{2}+n^{2}\right)^{2} \\
\left.+\frac{m n \pi^{2} \bar{D}_{1} B_{a}^{2}}{16 B_{h}^{4}} \bar{\xi}_{m n}\left[B_{a}^{2} K_{1}+\pi^{2}\left(m^{2} B_{a}^{2}+n^{2}\right) K_{2}\right]\right\} \bar{W} \\
+\frac{\bar{E}_{1} m n \pi^{6}}{256 B_{h}^{4}}\left(m^{4} B_{a}^{4}+n^{4}\right) \bar{\xi}_{m n} \bar{W}(\bar{W}+\mu)(\bar{W}+2 \mu) \\
+\frac{m n \pi^{4}}{16 B_{h}^{2}} \bar{\xi}_{m n}\left(m^{2} B_{a}^{2} \bar{N}_{x 0}+n^{2} \bar{N}_{y 0}\right)(\bar{W}+\mu)=0,
\end{gathered}
$$

where $m, n$ are odd numbers, and

$$
\begin{aligned}
& \bar{E}_{i}=E_{i} / h^{i}(i=1 \div 7), \bar{W}=W / h, B_{a}=b / a, B_{h}=b / h, \bar{D}_{1}=D_{1} / h^{3}, \bar{D}_{2}=D_{2} / h^{5}, \\
& \bar{D}_{3}=D_{3} / h^{7}, \bar{D}_{4}=D_{4} / h^{3}, \bar{D}_{5}=D_{5} / h^{5}, \bar{D}_{6}=D_{6} / h, \bar{N}_{x 0}=N_{x 0} / h, \\
& \bar{N}_{y 0}=N_{y 0} / h, K_{1}=\frac{k_{1} a^{4}}{D_{1}}, K_{2}=\frac{k_{2} a^{2}}{D_{1}}, \bar{\zeta}_{m n}=\frac{\left(3 \bar{D}_{4}-4 \bar{D}_{5}\right) \pi^{2}}{3 \bar{D}_{6} B_{h}^{2}}\left(m^{2} B_{a}^{2}+n^{2}\right)+1 .
\end{aligned}
$$

In what follows, the fictitious compressive edge loads $N_{x 0}, N_{y 0}$ for the FGM plates under the tangential edge constraints will be specified. From Eqs. (6), (7) and (10) one can obtain the following relations in which Eq. (15) and imperfection have been included

$$
\begin{aligned}
& \frac{\partial u}{\partial x}=\frac{1}{E_{1}}\left(f_{, y y}-v f_{, x x}\right)-\frac{E_{2}}{E_{1}} \phi_{x, x}+\frac{c_{1} E_{4}}{E_{1}}\left(\phi_{x, x}+w_{, x x}\right)-\frac{1}{2} w_{, x}^{2}-w_{, x} w_{, x}^{*}+\frac{\Phi_{1}}{E_{1}}, \\
& \frac{\partial v}{\partial y}=\frac{1}{E_{1}}\left(f_{, x x}-v f_{, y y}\right)-\frac{E_{2}}{E_{1}} \phi_{y, y}+\frac{c_{1} E_{4}}{E_{1}}\left(\phi_{y, y}+w_{, y y}\right)-\frac{1}{2} w_{, y}^{2}-w_{, y} w_{, y}^{*}+\frac{\Phi_{1}}{E_{1}} .
\end{aligned}
$$

Introduction of Eqs. (20), (21) and (22) into Eqs. (28) and then substitution of the resulting equations into Eqs. (19) yield the following expressions

$$
\begin{aligned}
& \Delta_{1}=\frac{1}{E_{1}}\left(v N_{y 0}-N_{x 0}\right)-\frac{4 E_{2} B_{1} \beta_{m}}{m n \pi^{2} E_{1}}+\frac{4 c_{1} E_{4} \beta_{m}}{m n \pi^{2} E_{1}}\left(B_{1}+\beta_{m} W\right)+\frac{\beta_{m}^{2}}{8} W(W+2 \mu h)-\frac{\Phi_{1}}{E_{1}}, \\
& \Delta_{2}=\frac{1}{E_{1}}\left(v N_{x 0}-N_{y 0}\right)-\frac{4 E_{2} B_{2} \delta_{n}}{m n \pi^{2} E_{1}}+\frac{4 c_{1} E_{4} \delta_{n}}{m n \pi^{2} E_{1}}\left(B_{2}+\delta_{n} W\right)+\frac{\delta_{n}^{2}}{8} W(W+2 \mu h)-\frac{\Phi_{1}}{E_{1}} .
\end{aligned}
$$

Introduction of Eqs. (29) into Eqs. (17) and (18) and solving obtained equations, the fictitious compressive edge loads can be determined as

$$
\begin{aligned}
& \bar{N}_{x 0}=e_{11} \bar{W}+e_{12} \bar{W}(\bar{W}+2 \mu)-e_{13} \Phi_{1} / h, \\
& \bar{N}_{y 0}=e_{21} \bar{W}+e_{22} \bar{W}(\bar{W}+2 \mu)-e_{23} \Phi_{1} / h,
\end{aligned}
$$


where

$$
\begin{aligned}
e_{11}= & -\frac{4 e \bar{E}_{2}}{m n \pi B_{h}}\left[\bar{s}_{1}\left(\bar{E}_{1}+\bar{s}_{2}\right) m B_{a} \bar{B}_{1}+v \bar{s}_{1} \bar{s}_{2} n \bar{B}_{2}\right] \\
& +\frac{16 e \bar{E}_{4}}{3 m n \pi B_{h}^{2}}\left[\bar{s}_{1}\left(\bar{E}_{1}+\bar{s}_{2}\right)\left(m B_{a} B_{h} \bar{B}_{1}+\pi m^{2} B_{a}^{2}\right)+v \bar{s}_{1} \bar{s}_{2}\left(n B_{h} \bar{B}_{2}+\pi n^{2}\right)\right], \\
e_{12}= & \frac{\pi^{2} e \bar{E}_{1}}{8 B_{h}^{2}}\left[\bar{s}_{1}\left(\bar{E}_{1}+\bar{s}_{2}\right) m^{2} B_{a}^{2}+v n^{2} \bar{s}_{1} \bar{s}_{2}\right], \quad e_{13}=e\left[v \bar{s}_{1} \bar{s}_{2}+\bar{s}_{1}\left(\bar{E}_{1}+\bar{s}_{2}\right)\right], \\
e_{21}= & -\frac{4 e \bar{E}_{2}}{m n \pi B_{h}^{2}}\left[\bar{s}_{2}\left(\bar{E}_{1}+\bar{s}_{1}\right) n B_{h} \bar{B}_{2}+v \bar{s}_{1} \bar{s}_{2} m B_{a} B_{h} \bar{B}_{1}\right] \\
& +\frac{16 e \bar{E}_{4}}{3 \pi m n B_{h}^{2}}\left[\bar{s}_{2}\left(\bar{E}_{1}+\bar{s}_{1}\right)\left(n B_{h} \bar{B}_{2}+\pi n^{2}\right)+v \bar{s}_{1} \bar{s}_{2}\left(m B_{a} B_{h} \bar{B}_{1}+\pi m^{2} B_{a}^{2}\right)\right], \\
e_{22}= & \frac{\pi^{2} e \bar{E}_{1}}{8 B_{h}^{2}}\left[v \bar{s}_{1} \bar{s}_{2} m^{2} B_{a}^{2}+\bar{s}_{2}\left(\bar{E}_{1}+\bar{s}_{1}\right) n^{2}\right], \quad e_{23}=e\left[v \bar{s}_{1} \bar{s}_{2}+\bar{s}_{2}\left(\bar{E}_{1}+\bar{s}_{1}\right)\right],
\end{aligned}
$$

in which

$$
\bar{s}_{1}=s_{1} / h, \quad \bar{s}_{2}=s_{2} / h, \quad e=\frac{1}{\left(\bar{E}_{1}+\bar{s}_{1}\right)\left(\bar{E}_{1}+\bar{s}_{2}\right)-v^{2} \bar{s}_{1} \bar{s}_{2}},
$$

and $\bar{B}_{1}, \bar{B}_{2}$ are analogous to those given in the [14].

In this study, the FGM plate is entirely exposed to thermal environments uniformly raised from thermal stress free initial state $T_{0}$ to value $T$ and temperature change $\Delta T=$ $T-T_{0}$ is considered to be independent of thickness variable $z$. The thermal expression $\Phi_{1}$ is obtained from Eqs. (11) as

$$
\Phi_{1} / h=H(T) \Delta T, \quad H(T)=E_{c}(T) \alpha_{c}(T)+\frac{E_{c}(T) \alpha_{m c}(T)+E_{m c}(T) \alpha_{c}(T)}{N+1}+\frac{E_{m c}(T) \alpha_{m c}(T)}{2 N+1} .
$$

\section{STABILITY ANALYSIS}

\subsection{FGM plate under uniform temperature rise}

Introduction of Eqs. (35) into Eqs. (30), (31) and then substitution of the results into Eq. (26) give the following relation

$$
\Delta T=\frac{16 B_{h}^{2}}{m n \pi^{4} \bar{\zeta}_{m n}\left(m^{2} B_{a}^{2} e_{13}+n^{2} e_{23}\right) H(T)}\left[b_{11} \frac{\bar{W}}{\bar{W}+\mu}+b_{21} \bar{W}+b_{31} \bar{W}(\bar{W}+2 \mu)\right],
$$

where

$$
\begin{aligned}
b_{11}= & -\frac{\left(\bar{D}_{2} \bar{D}_{5}-\bar{D}_{3} \bar{D}_{4}\right)}{9 \bar{D}_{6} B_{h}^{6}} m n \pi^{8}\left(m^{2} B_{a}^{2}+n^{2}\right)^{3}+\frac{m n \pi^{6}}{16 B_{h}^{4}}\left(\frac{4}{3} \bar{D}_{2}+\bar{D}_{4}\right)\left(m^{2} B_{a}^{2}+n^{2}\right)^{2} \\
& +\frac{m n \pi^{2} \bar{D}_{1} B_{a}^{2}}{16 B_{h}^{4}} \bar{\xi}_{m n}\left[B_{a}^{2} K_{1}+\pi^{2}\left(m^{2} B_{a}^{2}+n^{2}\right) K_{2}\right],
\end{aligned}
$$




$$
\begin{aligned}
& b_{21}=\frac{m n \pi^{4}}{16 B_{h}^{2}} \bar{\xi}_{m n}\left(m^{2} B_{a}^{2} e_{11}+n^{2} e_{21}\right) \\
& b_{31}=\frac{\bar{E}_{1} m n \pi^{6}}{256 B_{h}^{4}}\left(m^{4} B_{a}^{4}+n^{4}\right) \bar{\xi}_{m n}+\frac{m n \pi^{4}}{16 B_{h}^{2}} \bar{\xi}_{m n}\left(m^{2} B_{a}^{2} e_{12}+n^{2} e_{22}\right) .
\end{aligned}
$$

It is evident from Eq. (36) that bifurcation type buckling behavior can occur for geometrically perfect FGM plates (i.e. $\mu=0$ ) and buckling temperature change of thick FGM rectangular plates accounting for effects of elastic foundations and tangential restraints of edges can be predicted as

$$
\Delta T_{b}=\frac{16 B_{h}^{2} b_{11}}{m n \pi^{4} \bar{\zeta}_{m n}\left(m^{2} B_{a}^{2} e_{13}+n^{2} e_{23}\right) H(T)} .
$$

Eqs. (36) and (38) are explicit expressions of temperature-deflection relation and buckling temperature change for FGM plates in case of material properties are temperature independent. In contrast, as temperature dependence of material properties is included, Eqs. (36) and (38) are implicit expressions and an iteration algorithm must be utilized to obtain critical buckling temperatures and postbuckling temperature-deflection curves. Detailed procedure of iteration process is similar as that suggested in the work [25] and is omitted here for sake of brevity. The error tolerance of iteration in the present study is 0.001 .

\subsection{FGM plate under uniform uniaxial compression in a thermal environment}

Consider a thick FGM plate resting on an elastic foundation and exposed to thermal environment. Simultaneously, the plate is subjected to uniaxial compressive load $F$ uniformly distributed on two edges $x=0, a$ assumed to be freely movable, whereas two unloaded edges $y=0, b$ is tangentially restrained. In this case, $\bar{N}_{x 0}=-F[14,24]$ and $\bar{N}_{y 0}$ is determined by following the same procedure described in the previous section as

$$
\bar{N}_{y 0}=e_{31} \bar{N}_{x 0}+e_{32} \bar{W}+e_{33} \bar{W}(\bar{W}+2 \mu)-e_{34} H(T) \Delta T
$$

where

$$
e_{34}=\frac{\bar{s}_{2}}{\bar{E}_{1}+\bar{s}_{2}}, e_{31}=v e_{34}, e_{33}=\frac{n^{2} \pi^{2} \bar{E}_{1}}{8 B_{h}^{2}} e_{34}, e_{32}=e_{34}\left[\frac{16 \bar{E}_{4}}{3 m \pi B_{h}}\left(\bar{B}_{2}+\frac{n \pi}{B_{h}}\right)-\frac{4 \bar{E}_{2} \bar{B}_{2}}{m \pi B_{h}}\right] .
$$

Substitution of $\bar{N}_{x 0}, \bar{N}_{y 0}$ into Eq. (26) leads to the following expression

$$
F=\frac{16 B_{h}^{2}}{m n \pi^{4} \bar{\zeta}_{m n}\left(m^{2} B_{a}^{2}+n^{2} e_{31}\right)}\left[b_{12} \frac{\bar{W}}{\bar{W}+\mu}+b_{22} \bar{W}+b_{32} \bar{W}(\bar{W}+2 \mu)-b_{42} \Delta T\right]
$$


where

$$
\begin{aligned}
b_{12}= & -\frac{\left(\bar{D}_{2} \bar{D}_{5}-\bar{D}_{3} \bar{D}_{4}\right)}{9 \bar{D}_{6} B_{h}^{6}} m n \pi^{8}\left(m^{2} B_{a}^{2}+n^{2}\right)^{3}+\frac{m n \pi^{6}}{16 B_{h}^{4}}\left(\frac{4}{3} \bar{D}_{2}+\bar{D}_{4}\right)\left(m^{2} B_{a}^{2}+n^{2}\right)^{2} \\
& +\frac{m n \pi^{2} \bar{D}_{1} B_{a}^{2}}{16 B_{h}^{4}} \bar{\xi}_{m n}\left[B_{a}^{2} K_{1}+\pi^{2}\left(m^{2} B_{a}^{2}+n^{2}\right) K_{2}\right], \quad b_{22}=\frac{m n \pi^{4}}{16 B_{h}^{2}} \bar{\xi}_{m n} n^{2} e_{32}, \\
b_{32}= & \frac{\bar{E}_{1} m n \pi^{6}}{256 B_{h}^{4}}\left(m^{4} B_{a}^{4}+n^{4}\right) \bar{\xi}_{m n}+\frac{m n \pi^{4}}{16 B_{h}^{2}} \bar{\xi}_{m n} n^{2} e_{33}, \quad b_{42}=\frac{m n \pi^{4}}{16 B_{h}^{2}} \bar{\xi}_{m n} n^{2} e_{34} H(T),
\end{aligned}
$$

It is observed from Eq. (41) that geometrically perfect FGM plates exhibit a bifurcation type buckling behavior with corresponding buckling compressive load is predicted as

$$
F_{b}=\frac{16 B_{h}^{2}}{m n \pi^{4} \bar{\zeta}_{m n}\left(m^{2} B_{a}^{2}+n^{2} e_{31}\right)}\left(b_{12}-b_{42} \Delta T\right) .
$$

To measure the degree of edge constraint in a more convenient way, alternate tangential stiffness parameters $\lambda_{1}$ and $\lambda_{2}$ are introduced such that $\lambda_{1}=0$ and $\lambda_{1}=1$ correspond to movable and immovable edges at $x=0$ and $a$, respectively. Similarly, $\lambda_{2}=0$ and $\lambda_{2}=1$ correspond to movable and immovable edges at $y=0$ and $b$, respectively. Partially restrained edges at $x=0, a$ and $y=0, b$ are defined by $0<\lambda_{1}<1$ and $0<\lambda_{2}<1$, respectively. In the present study, these alternate tangential stiffness parameters are defined by

$$
\lambda_{1}=\frac{\bar{s}_{1}}{\bar{E}_{1}\left(T_{0}\right)+\bar{s}_{1}}, \quad \lambda_{2}=\frac{\bar{s}_{2}}{\bar{E}_{1}\left(T_{0}\right)+\bar{s}_{2}},
$$

in which $\bar{E}_{1}\left(T_{0}\right)$ is value of $\bar{E}_{1}$ calculated at room temperature $T_{0}$.

\section{RESULTS AND DISCUSSION}

Towards the major purpose of the present study, this section presents numerical results for square plates $(a=b)$ made of functionally graded materials and for deformation modes with half wave numbers $m=n=1$.

As an example for verification of the present method, a simply supported perfect FGM rectangular plate without foundation interaction and exposed to uniform temperature rise is considered. The plate is immovable at all edges (i.e. $\lambda_{1}=\lambda_{2}=1$ ) and made of Aluminum $(\mathrm{Al})$ and Alumina $\left(\mathrm{Al}_{2} \mathrm{O}_{3}\right)$ with temperature independent material properties are $E_{m}=70 \mathrm{GPa}, \alpha_{m}=23 \times 10^{-6}\left({ }^{\circ} \mathrm{C}^{-1}\right)$ for $\mathrm{Al}$ and $E_{c}=380 \mathrm{GPa}, \alpha_{c}=7.4 \times 10^{-6}\left({ }^{\circ} \mathrm{C}^{-1}\right)$ for $\mathrm{Al}_{2} \mathrm{O}_{3}$, whereas $v=0.3$ for both constituents. Critical buckling temperature change $\Delta T_{c r}$ for $\mathrm{Al} / \mathrm{Al}_{2} \mathrm{O}_{3} \mathrm{FGM}$ plates under uniform temperature rise is calculated by Eq. (38) and presented in Tab. 1 in comparison with results of Javaheri and Eslami [3] using adjacent equilibrium criterion in linear buckling analysis and results obtained by Loc et al. [9] making use of an isogeometric finite element formulation for thermal buckling analysis. In this table $N^{*}$ is volume fraction index for case of $V_{c}(z)=(z / h+1 / 2)^{N^{*}}$. As can be seen, an excellent agreement is achieved in this comparison.

The remainder of this section presents numerical results for FGM rectangular plates composed of silicon nitride $\left(\mathrm{Si}_{3} \mathrm{~N}_{4}\right)$ and stainless steel (SUS304). The material properties 
Hoang Van Tung

Table 1. Critical buckling temperature difference $\Delta T_{c r}$ of perfect $\mathrm{Al} / \mathrm{Al}_{2} \mathrm{O}_{3}$ FGM square plates under uniform temperature rise

\begin{tabular}{|c|c|c|c|c|c|c|c|}
\hline \multirow{2}{*}{$b / h$} & \multirow{2}{*}{ Source } & \multicolumn{7}{|c|}{ Power index $N^{*}$} \\
\cline { 3 - 8 } & & 0 & 0.5 & 1 & 4 & 5 & 10 \\
\hline 10 & Ref. [3] & 1617.484 & - & 757.891 & - & 678.926 & 692.519 \\
\cline { 2 - 8 } & Ref. [9] & 1618.7468 & 923.1991 & 758.4268 & 670.4594 & 679.3379 & 692.7225 \\
\cline { 2 - 8 } & Present & 1618.6819 & 923.1617 & 758.3956 & 670.4320 & 679.3104 & 692.6948 \\
\hline \multirow{2}{*}{20} & Ref. [3] & 421.516 & - & 196.257 & - & 178.528 & 183.141 \\
\cline { 2 - 8 } & Present & 421.535 & 239.2399 & 196.265 & 175.574 & 178.535 & 183.144 \\
\hline \multirow{2}{*}{40} & Ref. [3] & 106.492 & - & 49.500 & - & 45.213 & 46.455 \\
\cline { 2 - 8 } & Present & 106.494 & 60.363 & 49.502 & 44.422 & 45.214 & 46.455 \\
\hline
\end{tabular}

Table 2. Temperature-dependent thermo-elastic coefficients for silicon nitride and stainless steel (Reddy and Chin [27])

\begin{tabular}{|c|c|c|c|c|c|c|}
\hline Materials & Properties & $P_{0}$ & $P_{-1}$ & $P_{1}$ & $P_{2}$ & $P_{3}$ \\
\hline Silicon nitride & $E(\mathrm{~Pa})$ & $348.43 \mathrm{e}+9$ & 0 & $-3.070 \mathrm{e}-4$ & $2.160 \mathrm{e}-7$ & $-8.946 \mathrm{e}-11$ \\
\cline { 2 - 7 } & $\alpha(1 / \mathrm{K})$ & $5.8723 \mathrm{e}-6$ & 0 & $9.095 \mathrm{e}-4$ & 0 & 0 \\
\hline \multirow{3}{*}{ Stainless steel } & $E(\mathrm{~Pa})$ & $201.04 \mathrm{e}+9$ & 0 & $3.079 \mathrm{e}-4$ & $-6.534 \mathrm{e}-7$ & 0 \\
\cline { 2 - 7 } & $\alpha(1 / \mathrm{K})$ & $12.330 \mathrm{e}-6$ & 0 & $8.086 \mathrm{e}-4$ & 0 & 0 \\
\hline
\end{tabular}

$\operatorname{Pr}$, such as elasticity modulus $E$ and thermal expansion coefficient $\alpha$ can be expressed as a nonlinear function of temperature [28]

$$
\operatorname{Pr}=P_{0}\left(P_{-1} T^{-1}+1+P_{1} T+P_{2} T^{2}+P_{3} T^{3}\right),
$$

in which $T=T_{0}+\Delta T$ and $T_{0}=300 \mathrm{~K}$ (room temperature), $P_{0}, P_{-1}, P_{1}, P_{2}$ and $P_{3}$ are the coefficients of temperature $T(K)$ and are unique to the constituent materials. Specific values of these coefficients for $E$ and $\alpha$ of silicon nitride and stainless steel are given by Reddy and Chin [27] and are listed in Tab. 2. Poisson's ratio is assumed to be a constant $v=0.3$. In addition, temperature-dependent and temperature-independent material properties will be written as T-D and T-ID, respectively, for sake of brevity. The T-ID are material properties calculated at room temperature $T_{0}=300 \mathrm{~K}$. Tab. 3 indicates that both critical buckling temperature and difference between buckling temperatures in two cases T-D and T-ID are increased as FGM plates become thicker and/or ceramic rich.

Tab. 4 shows the effects of the stiffness parameters of elastic foundation and the degree of edge constraint on the critical buckling temperatures of perfect FGM plates under uniform temperature rise. As can be seen, the critical buckling temperatures are enhanced as the stiffness parameters $K_{1}, K_{2}$ of foundation are increased and the degree of tangential edge constraint $\lambda_{1}, \lambda_{2}$ are reduced. Moreover, the effects of temperature dependent material properties on critical buckling temperatures become more pronounced 
Table 3. Effects of power index $N$ and $b / h$ ratio on the critical buckling temperatures $T_{c r}=T_{0}+\Delta T_{c r}(\mathrm{~K})$ for immovable FGM plates $\left(a / b=1, K_{1}=K_{2}=0, \lambda_{1}=\lambda_{2}=1, T_{0}=300 \mathrm{~K}\right)$

\begin{tabular}{|c|c|c|c|c|c|c|}
\hline \multirow{2}{*}{$N$} & & \multicolumn{5}{|c|}{$b / h$} \\
\cline { 2 - 7 } & 10 & 15 & 20 & 25 & 30 & 40 \\
\hline 0 & $866^{a}\left(1082^{b}\right)$ & $598(658)$ & $481(504)$ & $421(431)$ & $386(391)$ & $350(351)$ \\
\hline 0.5 & $964(1263)$ & $655(741)$ & $518(551)$ & $446(461)$ & $405(412)$ & $361(363)$ \\
\hline 1 & $1005(1362)$ & $683(786)$ & $537(576)$ & $459(478)$ & $414(424)$ & $367(370)$ \\
\hline 2 & $1049(1475)$ & $713(837)$ & $557(605)$ & $474(496)$ & $425(437)$ & $373(377)$ \\
\hline 5 & $1111(1627)$ & $754(907)$ & $585(645)$ & $494(522)$ & $440(454)$ & $382(387)$ \\
\hline 10 & $1157(1727)$ & $780(952)$ & $603(671)$ & $507(538)$ & $419(466)$ & $388(394)$ \\
\hline 100 & $1234(1879)$ & $822(1023)$ & $630(711)$ & $526(564)$ & $464(484)$ & $397(404)$ \\
\hline
\end{tabular}

${ }^{a} \mathrm{~T}-\mathrm{D},{ }^{b} \mathrm{~T}-\mathrm{ID}$

and deteriorative for FGM plates of which boundary edges are partially restrained in tangential direction and/or are supported by stiffer elastic foundations, i.e. with higher values of parameters $K_{1}, K_{2}$, especially Pasternak type foundations. As degree of tangential constraint is increased, i.e. higher values of parameters $\lambda_{1}, \lambda_{2}$, difference between critical buckling temperatures for T-D and T-ID cases is smaller. These are graphically illustrated in Fig. 2.

Table 4. Effects of elastic foundations and degree of edge constraints on critical buckling temperatures of FGM plates $T_{c r}=T_{0}+\Delta T_{c r}(\mathrm{~K})\left(a / b=1, b / h=20, N=2, T_{0}=300 \mathrm{~K}\right)$

\begin{tabular}{|c|c|c|c|c|c|}
\hline \multirow{2}{*}{$K_{1}, K_{2}$} & \multicolumn{5}{|c|}{$\lambda_{1}, \lambda_{2}$} \\
\cline { 2 - 6 } & $0.4,0.4$ & $0.5,0.5$ & $0.6,0.6$ & $0.8,0.8$ & 1,1 \\
\hline 0,0 & $900^{a}\left(1260^{b}\right)$ & $805(1042)$ & $733(896)$ & $629(714)$ & $557(605)$ \\
\hline 50,0 & $951(1385)$ & $852(1138)$ & $775(974)$ & $663(768)$ & $586(645)$ \\
\hline 50,10 & $1118(1877)$ & $1009(1519)$ & $921(1280)$ & $788(981)$ & $691(802)$ \\
\hline 50,20 & $1244(2370)$ & $1136(1900)$ & $1043(1586)$ & $897(1194)$ & $786(959)$ \\
\hline 100,10 & $1153(2002)$ & $1044(1615)$ & $954(1357)$ & $817(1035)$ & $716(841)$ \\
\hline
\end{tabular}

${ }^{a} \mathrm{~T}-\mathrm{D},{ }^{b} \mathrm{~T}-\mathrm{ID}$

The effects of tangential edge constraints and elastic foundations on the thermal postbuckling behavior of geometrically perfect FGM plates are analyzed in Figs. 3 and 4. Fig. 3 shows the effects of tangential constraint of edges on the thermal postbuckling of FGM plates without foundation interaction, i.e. $K_{1}=K_{2}=0$, in T-D case. As can be observed, increase in the degree of edge constraint has pronounced and deteriorative 
influences on both critical buckling temperature and postbuckling loading capacity of thermally loaded FGM plates.

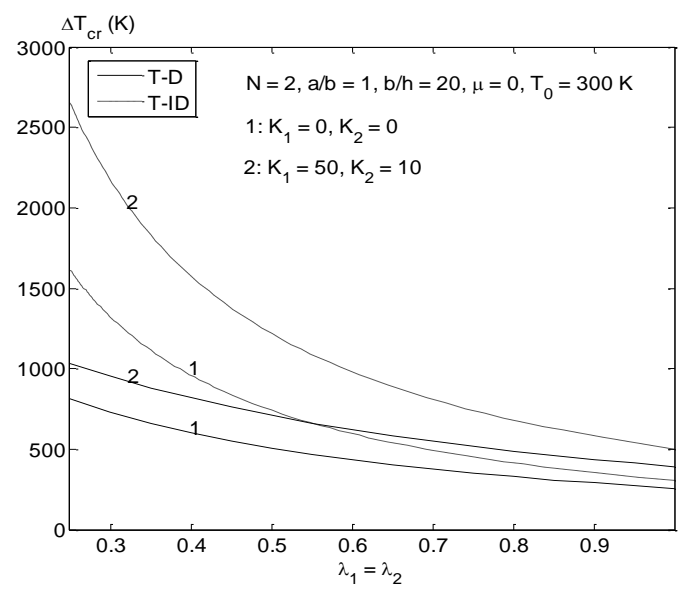

Fig. 2. Effects of tangential edge constraints and elastic foundations on the buckling temperatures of perfect FGM plates

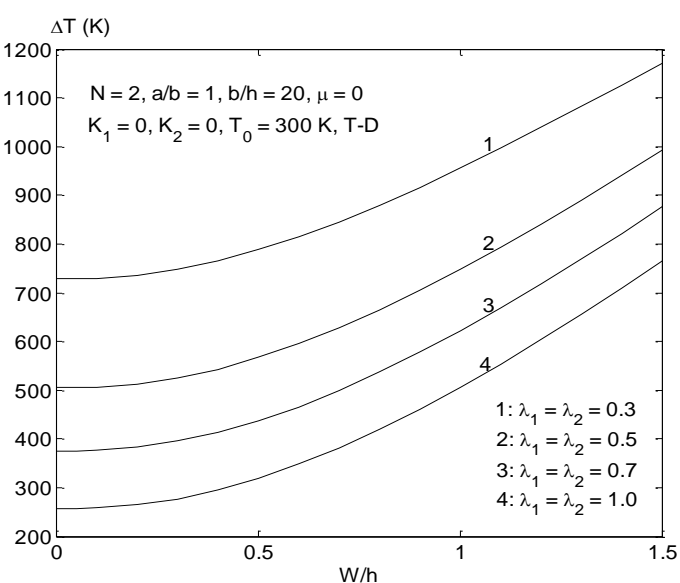

Fig. 3. Effects of edge constraints on the thermal postbuckling of FGM plates without elastic foundations and T-D properties

The simultaneous influences of tangential edge restraint, elastic foundations and temperature dependent material properties on the thermal postbuckling of perfect FGM plates are depicted in Fig. 4.

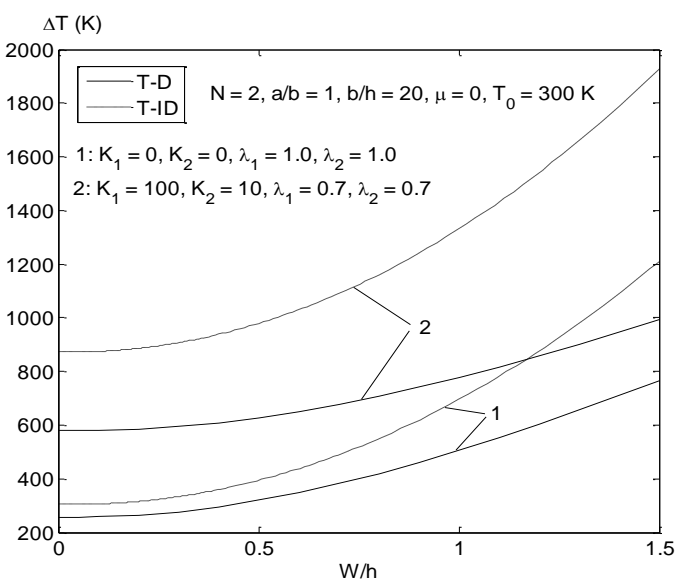

Fig. 4. Combined effects of edge constraints, elastic foundations and T-D properties on the thermal postbuckling of FGM plates

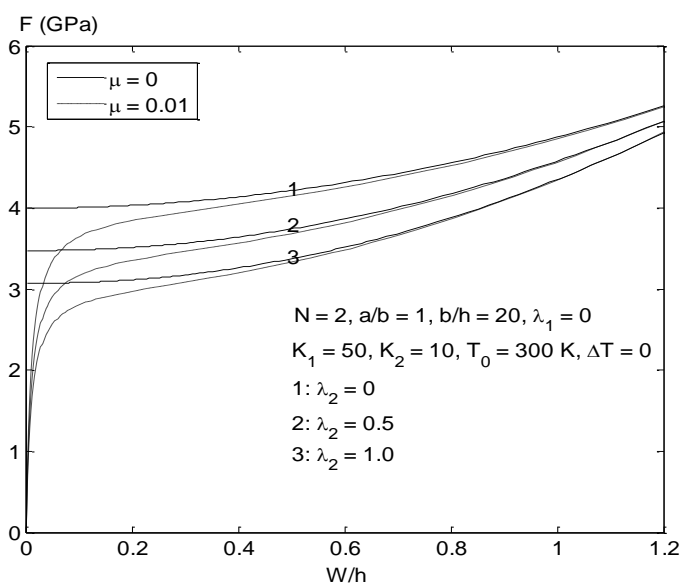

Fig. 5. Effects of edge constraint at edges $y=$ $0, b$ on the postbuckling of FGM plates under uniform uniaxial compression

As shown, the difference between load-deflection curves in T-D and T-ID cases is smaller for immovable edge FGM plates without the foundation interaction. In contrast, there is the existence of a sharp difference between postbuckling equilibrium paths 
of partially restrained edge FGM plates resting on elastic foundations for two T-D and T-ID cases. Fig. 5 illustrates the effects of degree of tangential constraint at unloaded edges $y=0, b$ on the mechanical postbuckling behavior of FGM plates resting on an elastic foundation and subjected to uniform uniaxial compression at room temperature, i.e. $\Delta T=0$.

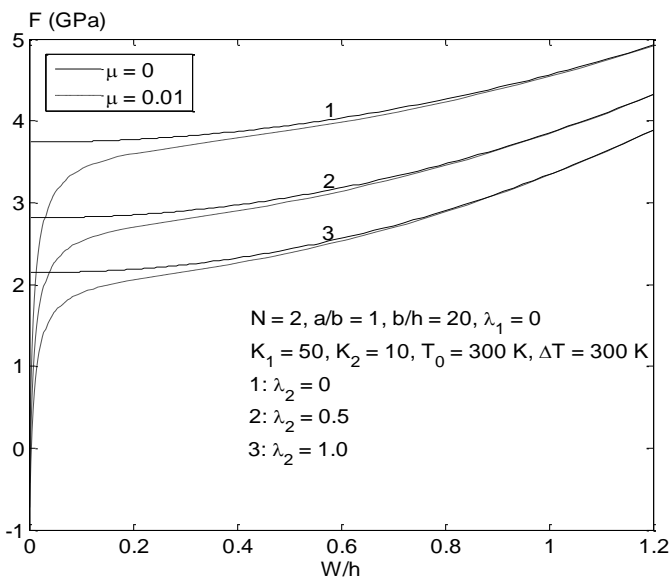

Fig. 6. Effects of constraint of edges $y=0, b$ on the thermomechanical postbuckling

Finally, Fig. 6 plotted as a counterpart of Fig. 5 for case of $\Delta T=300 \mathrm{~K}$ considers the effects of $\lambda_{2}$ parameter on the thermomechanical postbuckling of mechanically compressed FGM plates resting on an elastic foundation and exposed to a thermal environment. Obviously, critical buckling compressive loads and postbuckling strength are remarkably decreased as unloaded edges $y=0, b$ are more rigorously restrained, especially at elevated temperature.

\section{CONCLUDING REMARKS}

The thermal and thermomechanical postbuckling behavior of thick FGM plates resting on elastic foundations with tangentially restrained edges have been analyzed. The analysis reveals that the tangential constraints of boundary edges has extremely sensitive influences on the buckling and postbuckling of FGM plates. Specifically, critical buckling loads and postbuckling load capacity of FGM plates are decreased due to the rigorous constraint in tangential motion of edges. The results also shows that deteriorative effects of temperature dependent material properties on the thermal buckling and postbuckling behavior of thick FGM plates are more pronounced as FGM plates are ceramic-rich, rested on stiffer foundations and/or with lower degree of tangential edge constraint. Accordingly, temperature dependence of material properties must be considered for accurate predition of postbuckling behavior of thick FGM plates at highly elevated temperatures. 
Hoang Van Tung

\section{ACKNOWLEDGEMENT}

This research is funded by Vietnam National Foundation for Science and Technology Development (NAFOSTED) under grant number 107.02-2014.09.

\section{REFERENCES}

[1] R. Javaheri and M. R. Eslami. Buckling of functionally graded plates under in-plane compressive loading. ZAMM-Journal of Applied Mathematics and Mechanics/Zeitschrift für Angewandte Mathematik und Mechanik, 82, (4), (2002), pp. 277-283.

[2] R. Javaheri and M. R. Eslami. Thermal buckling of functionally graded plates. AIAA Journal, 40, (1), (2002), pp. 162-169.

[3] R. Javaheri and M. R. Eslami. Thermal buckling of functionally graded plates based on higher order theory. Journal of Thermal Stresses, 25, (7), (2002), pp. 603-625.

[4] B. A. S. Shariat and M. R. Eslami. Buckling of thick functionally graded plates under mechanical and thermal loads. Composite Structures, 78, (3), (2007), pp. 433-439.

[5] W. Lanhe. Thermal buckling of a simply supported moderately thick rectangular FGM plate. Composite Structures, 64, (2), (2004), pp. 211-218.

[6] X. Zhao, Y. Y. Lee, and K. M. Liew. Mechanical and thermal buckling analysis of functionally graded plates. Composite Structures, 90, (2), (2009), pp. 161-171.

[7] H. Nguyen-Xuan, L. V. Tran, T. Nguyen-Thoi, and H. C. Vu-Do. Analysis of functionally graded plates using an edge-based smoothed finite element method. Composite Structures, 93, (11), (2011), pp. 3019-3039.

[8] H. Nguyen-Xuan, L. V. Tran, C. H. Thai, and T. Nguyen-Thoi. Analysis of functionally graded plates by an efficient finite element method with node-based strain smoothing. Thin-Walled Structures, 54, (2012), pp. 1-18.

[9] L. V. Tran, C. H. Thai, and H. Nguyen-Xuan. An isogeometric finite element formulation for thermal buckling analysis of functionally graded plates. Finite Elements in Analysis and Design, 73, (2013), pp. 65-76.

[10] L. W. Zhang, P. Zhu, and K. M. Liew. Thermal buckling of functionally graded plates using a local Kriging meshless method. Composite Structures, 108, (2014), pp. 472-492.

[11] X. Zhao and K. M. Liew. Geometrically nonlinear analysis of functionally graded plates using the element-free kp-Ritz method. Computer Methods in Applied Mechanics and Engineering, 198, (33), (2009), pp. 2796-2811.

[12] P. Zhu, L. W. Zhang, and K. M. Liew. Geometrically nonlinear thermomechanical analysis of moderately thick functionally graded plates using a local Petrov-Galerkin approach with moving Kriging interpolation. Composite Structures, 107, (2014), pp. 298-314.

[13] H. V. Tung and N. D. Duc. Nonlinear analysis of stability for functionally graded plates under mechanical and thermal loads. Composite Structures, 92, (5), (2010), pp. 1184-1191.

[14] N. D. Duc and H. V. Tung. Mechanical and thermal postbuckling of higher order shear deformable functionally graded plates on elastic foundations. Composite Structures, 93, (11), (2011), pp. 2874-2881.

[15] J. Woo, S. A. Meguid, J. C. Stranart, and K. M. Liew. Thermomechanical postbuckling analysis of moderately thick functionally graded plates and shallow shells. International Journal of Mechanical Sciences, 47, (8), (2005), pp. 1147-1171.

[16] H.-S. Shen. Thermal postbuckling behavior of shear deformable FGM plates with temperature-dependent properties. International Journal of Mechanical Sciences, 49, (4), (2007), pp. $466-478$. 
[17] Y. Y. Lee, X. Zhao, and J. N. Reddy. Postbuckling analysis of functionally graded plates subject to compressive and thermal loads. Computer Methods in Applied Mechanics and Engineering, 199, (25), (2010), pp. 1645-1653.

[18] A. M. Zenkour. A comprehensive analysis of functionally graded sandwich plates: Part 2-Buckling and free vibration. International Journal of Solids and Structures, 42, (18), (2005), pp. 5243-5258.

[19] A. M. Zenkour and M. Sobhy. Thermal buckling of various types of FGM sandwich plates. Composite Structures, 93, (1), (2010), pp. 93-102.

[20] H.-S. Shen and S.-R. Li. Postbuckling of sandwich plates with FGM face sheets and temperature-dependent properties. Composites Part B: Engineering, 39, (2), (2008), pp. 332344 .

[21] Z.-X. Wang and H.-S. Shen. Nonlinear analysis of sandwich plates with FGM face sheets resting on elastic foundations. Composite Structures, 93, (10), (2011), pp. 2521-2532.

[22] L. Librescu, W. Lin, M. P. Nemeth, and J. H. Starnes Jr. Thermomechanical postbuckling of geometrically imperfect flat and curved panels taking into account tangential edge constraints. Journal of Thermal Stresses, 18, (4), (1995), pp. 465-482.

[23] L. Librescu and W. Lin. Vibration of thermomechanically loaded flat and curved panels taking into account geometric imperfections and tangential edge restraints. International Journal of Solids and Structures, 34, (17), (1997), pp. 2161-2181.

[24] H. V. Tung. Postbuckling behavior of functionally graded cylindrical panels with tangential edge constraints and resting on elastic foundations. Composite Structures, 100, (2013), pp. 532 541.

[25] H. V. Tung. Postbuckling of functionally graded cylindrical shells with tangential edge restraints and temperature-dependent properties. Acta Mechanica, 225, (6), (2014), pp. 17951808.

[26] J. N. Reddy. Mechanics of laminated composite plates and shells: theory and analysis. CRC press, (2004).

[27] J. N. Reddy and C. D. Chin. Thermomechanical analysis of functionally graded cylinders and plates. Journal of Thermal Stresses, 21, (6), (1998), pp. 593-626.

[28] Y. S. Touloukian. Thermophysical properties of high temperature solid materials. New York MacMillan, (1967). 